\title{
Surface Gradient Approach for Occlusion Detection Based on Triangulated Irregular Network for True Orthophoto Generation
}

\author{
Henrique Cândido de Oliveira ${ }^{(\mathbb{0})}$, Aluir Porfírio Dal Poz ${ }^{\circledR}$, Mauricio Galo ${ }^{\mathbb{D}}$, and Ayman Fawzy Habib
}

\begin{abstract}
Aerial images of urban areas have been used as base information for a diversity of applications. Considering the great quantity of tall buildings in these areas, it is important to have a method to automatically generate a product called true orthophoto mosaic, which represents all objects above the ground (buildings, bridges, etc.) in their true location. However, to create a true orthophoto, it is necessary to consider the occlusions caused by the surface height variation and to compensate for the lack of information using adjacent aerial images. The automatic occlusion detection is the bottleneck during the true orthophoto mosaic generation. The main aim of this paper is to introduce a new approach for occlusion detection - the surface-gradient-based method (SGBM) applied to a triangulated irregular network (TIN) representation. The originality of the SGBM is the occlusion detection principle, which is based on the concept of surface gradient behavior analysis over a TIN surface. The current methods interpolate a point cloud into a gridded digital surface model, which can introduce artifacts to the representation. The SGBM represents the surface as a TIN-based solid by taking into account the Delaunay constraint in the original point cloud, avoiding the interpolation step. The occlusions are then compensated using specific cost functions and refined via color blending. Experiments were performed and the results were assessed by using quality indicators (completeness), the consistency of orthoimage mosaic, and the time of processing. Experimental results demonstrated the feasibility of the SGBM for occlusion detection in the true orthophoto generation.
\end{abstract}

Index Terms-Mosaicking, occlusion detection, point cloud, surface-gradient, TIN-based polyhedron, true orthophoto.

\section{INTRODUCTION}

I MAGE datasets obtained from aerial platforms, as well as the derivative products, have been used for decades as base information to take decisions in several applications. These aerial images are acquired in a perspective projection, which cause

Manuscript received May 29, 2017; revised September 25, 2017 and December 5, 2017; accepted December 11, 2017. Date of publication January 16, 2018; date of current version February 12, 2018. The work of H. C. de Oliveira was supported by the FAPESP (Fundação de Amparo à Pesquisa do Estado de São Paulo - Brazil) under Grant 2013/21647-2 and Grant 2014/15332-1. (Corresponding author: Henrique Cândido de Oliveira.)

H. C. de Oliveira is with the Faculty of Civil Engineering, Architecture, and Urban Planning, University of Campinas, Campinas 13083-889, Brazil (e-mail: oliveira@ fec.unicamp.br).

A. P. Dal Poz and M. Galo are with the Department of Cartography, São Paulo State University, Presidente Prudente 19060900, Brazil (e-mail: aluir@ fct.unesp.br; galo@fct.unesp.br).

A. F. Habib is with the Lyles School of Civil Engineering, Purdue University, West Lafayette, IN 47907-2050 USA (e-mail: ahabib@purdue.edu).

Color versions of one or more of the figures in this paper are available online at http://ieeexplore.iee.org.

Digital Object Identifier 10.1109/JSTARS.2017.2786162 the scale to be nonuniform. To extract precise information from these images, the use of an orthophoto is advised because it has uniform scale and can be considered geometrically equivalent to a line map [1].

However, the conventional orthorectification process [2] takes into account only the terrain surface - digital terrain model (DTM), and does not represent objects above the ground in orthogonal projection. Therefore, the height variation of structures of interest, such as buildings and bridges, are not considered during the orthorectification process, which is a problem when orthophotos need to be used in projects performed over dense urban areas. Buildings and other tall objects are not rectified correctly causing occluded areas - not visible from the perspective center (PC) of the camera. These occlusions can hide important features that may be essential for the project execution.

Bearing in mind the problems caused by occlusions, the orthorectification process needs to consider the elements above ground (buildings, bridges, trees, etc). Therefore, the surface representation must also contain structures that are not represented on the DTM - this representation is called digital surface model (DSM). On the other hand, the use of a DSM by itself during the orthorectification process does not guarantee the correct orthogonal location of the buildings and the proper occlusion compensation. Actually, the use of a DSM can introduce undesirable effects, such as the double mapping, also known as ghost image [3]. This effect is caused by the competition between two or more groundels (ground element, which corresponds to a DSM cell) for a single image pixel during the orthorectification process. This double mapping represents both, the correct location of the object (the roof of the building) and the occlusion area ("ghost image"). More explanation about the double mapping effect can be seen in [3] and [4].

An orthophoto that has uniform scale and is free from occlusions and double mapping is called "true orthophoto." In order to generate this product, it is necessary to detect and compensate for the occlusions by using adjacent images from the flight mission, selected by considering some criteria or a specific cost function, followed by a color adjustment procedure. The occlusion detection is the major bottleneck during the true orthophoto generation; therefore, this is the main focus of this paper.

Several methods used for this purpose can be found in the literature, as the Z-buffer (see [4], [5], and [9]); methods based on digital building model (DBM) (see [9]-[15]); the height-based ray tracing (see [3], [16], and [17]); the height-gradient-based 
method (see [18]-[20]); and the angle-based methods [4]. The main differences among them are the metric considered to determine the visibility between PC and DSM cells (if it is occluded or not), and the type of input information required to apply the occlusion detection. More complete explanations of the aforementioned methods, along with their characteristics, advantages, and disadvantages, are presented and discussed in Section II.

The main drawback of the mentioned methods is the use of a gridded-DSM to represent the surface, which requires an interpolation process for its generation (it adds more time to generate the true orthophoto). The interpolation can also result in undesirable artifacts into the DSM, such as sloping facades in vertical buildings, due to lack of information in the original point cloud.

To circumvent the drawbacks from the existing state-of-theart methods for the occlusion detection, especially for the ones that consider the interpolation of a DSM into a grid, a new approach for occlusion detection is proposed in this paper; the surface-gradient-based method (SGBM) using a TIN as a data structure to represent the surface. This method treats the terrain surface as a polyhedron, i. e., using the original point cloud - without an initial interpolation step. In order to allow a more complete evaluation of the proposed occlusion detection method, occlusion compensation is also addressed in this paper. Some previous works (see [33] and [34]) used a TIN-based structure, not for representing the original point cloud as this approach does, but for representing reconstructed buildings (i.e., DBMs in [34]) and to create triangle prism model in [33].

This paper is organized as follows. In Section II, the state of the art in occlusion detection is presented, together with main characteristics of existing methods and main contributions of the proposed method. Section III introduces the proposed method for occlusion detection, along with other necessary methods for a complete generation of true orthoimages. In Section IV, experiments are performed to validate the proposed method. Section $\mathrm{V}$ presents the main conclusions and recommendations for future work.

\section{RELATED WORK}

Several methods for occlusion detection can be found in the literature. These methods will be presented in this section, with special attention to their relevant characteristics. It is important to emphasize that the occlusions in the images always occur in radial directions from the image nadir point, due to the perspective projection in the image acquisition. Therefore, all methods analyze surface profiles defined at radial directions from the ground nadir point [1] to detect these occlusion areas. It is important to highlight the necessity for all methods of having the camera interior orientation parameters (IOPs): calibrated focal length and lens distortion parameters, and the exterior orientation parameters (EOPs): position and attitude of each image.

\section{A. Z-Buffer Method}

The Z-buffer method has its origin in computer graphics. It was applied for the first time to occlusion detection in aerial imagery by [5]. This method uses the distance between the camera PC and the groundel to verify the visibility, aiming to solve the problem of image pixel competition that causes the double mapping effect.

For every image pixel, the distance between the PC and the current groundel is stored. Whenever, during the orthophoto generation, a selected image pixel has already been used to assign the gray values for a pixel in the orthophoto, the distance between the correspondent groundel and the PC is compared to the previously stored distance. The groundel with the shortest distance is considered visible, and the image pixel (in competition) is used to interpolate the radiometric values at the correspondent pixel in the orthophoto. The occluded groundel, i.e., the one that is farthest from the camera PC, is stored in a visibility map, which has the same spatial resolution as the input DSM and the output orthophoto [4]. The visibility map is then utilized as a guide for occlusion compensation by using adjacent images.

Further explanations of the Z-buffer method or modification of it can be seen at [4]-[9].

\section{B. DBM-Based (Projective) Method}

The DBM-based method uses a DBM to represent the elements above the ground. The DBM generation is also performed by a preprocessing step and it can be derived using several approaches, such as the method presented by [10]. Assuming that one DBM is available, as far as the edges and ridges (lines) of a roof are known, it is possible to project them onto the ground level and detect the extent of the occlusion created by this building. Consequently, all pixels between the beginning and the end of the occlusion are labeled as occluded. For further explanations and results from DBM-based methods for occlusion detection, the references [9], [11]-[15], [33], and [34] are suggested.

\section{Height-Based Ray Tracing Method}

The height-based ray tracing approach was introduced by [16], as an alternative methodology for true orthophoto generation using satellite imagery. This method can be explained as follows: A given groundel $\left(\boldsymbol{g}_{u}\right)$ - extracted from a griddedDSM - is intended to be analyzed regarding its visibility in relation to the PC of the camera. First, the line of sight between the PC and the $\boldsymbol{g}_{u}$ is determined $\left(\boldsymbol{l}_{p}\right)$. The projection of $\boldsymbol{l}_{p}$ onto the DSM will give the groundels that represent the radial direction (search path). Starting from $\boldsymbol{g}_{u}$ along the search path, until the ground nadir point, all groundels are checked. If any of these groundels (on the search path-radial direction) has its altitude above the line $\boldsymbol{l}_{p}$, i.e., if its altitude is higher than $\boldsymbol{l}_{p}$, then $\boldsymbol{g}_{u}$ is labeled as occluded. A similar approach and additional details can be seen in [3] and [17].

\section{Height-Gradient-Based Method}

The height-gradient-based method [18] aims to solve the problem of image pixel competition (double mapping) by analyzing the height gradient of objects. Initially, a radial direction is determined over a gridded-DSM, by using Bresenham's line algorithm [19], starting at the ground nadir point and finishing 
at the image boundary projected onto the DSM. The height variation is calculated for all consecutive groundels in this direction (profile). The main idea is to identify the negative gradients that represent the beginning of an occlusion area in this direction. Once the negative gradient is found for one specific point in object space, it can be projected onto the image plane by using the collinearity equations [2]. This procedure derives the end of an occlusion for the current radial direction and consequently the occluded elements. A visibility map is then created by storing all occluded groundels, after analyzing all sampled radial directions. Additional explanation and results of the approach along with quality evaluation can be seen in [18]-[20]

\section{E. Angle-Based Method}

The angle-based method was presented by [4] to avoid problems posed by the Z-buffer method. The angle-based method uses an off-nadir angle to determine whether a groundel is occluded or visible. This angle is defined by the nadir direction and the line that connects the PC and a selected groundel. For each radial direction (starting at the ground nadir point), consecutive off-nadir angles are compared. The off-nadir angles tend to increase as the groundel is located further from the ground nadir point. However, when this angle decreases, it is an indication that an occlusion area begins. The succeeding groundels are labeled as occluded in the visibility map until the angle becomes greater than the angle corresponding to the last visible groundel. After a sweep of all radial directions, a visibility map is available, as in other methodologies.

Two different sweep strategies for defining the radial direction are also presented by [4]: the adaptive radial sweep method and the spiral sweep method. These different sweep strategies aim to avoid a great quantity of revisits to those groundels close to the ground nadir point. More details about the angle-based method and its variations can be seen in [4].

\section{F. Characteristics of the Existing Methods and Potential Contributions of the Method Proposed in This Paper}

As can be observed, the methods presented in this section have different metrics to detect occlusion areas and, as a consequence, different characteristics among them.

The Z-buffer method has the problem of false visibilities and occlusions caused by the following two main reasons: the difference between the ground sample distance (GSD) of the image and the gridded-DSM resolution, and tall-narrow buildings that are located in the mapping area [4]. Additional drawbacks are the necessity of a gridded-DSM to represent the surface.

The DBM-based method usually achieves better results when compared to methods based on only DSM analysis. It happens whenever roof edges are well defined, which can be a problem when using point clouds generated by using light detection and ranging (LiDAR) systems or dense image matching techniques. On the other hand, so far there is no fully automated DBM generation approach that is able to reliably extract the shape of all objects on a surface. Manual operator interference is usually necessary during the DBM generation. It has impact in the
TABLE I

CHARACTERISTICS OF THE REvised Methods

\begin{tabular}{lcccc}
\hline \hline Methods & \multicolumn{5}{c}{ Characteristics } \\
\cline { 2 - 5 } & (a) & (b) & (c) & (d) \\
\hline Z-buffer & $\mathrm{X}$ & - & - & $\mathrm{X}$ \\
DBM-based & - & - & $\mathrm{X}$ & - \\
Height-based ray tracing & $\mathrm{X}$ & $\mathrm{X}$ & - & - \\
Height-gradient-based & $\mathrm{X}$ & $\mathrm{X}$ & - & - \\
Angle-based & $\mathrm{X}$ & - & - & - \\
SGBM (proposed method) & - & $\mathrm{X}$ & - & - \\
\hline \hline
\end{tabular}

cost of true orthophoto generation and, in addition, might be a restrictive factor for a fast production of such product.

The height-based ray tracing method also needs a griddedDSM as a data source. It checks all groundels to identify whether it is occluding or not another groundel - that can be considered a disadvantage, due to the repetition of revisits to the same groundels several times.

The height-gradient-based method has the following main disadvantages: the use of a gridded-DSM to represent the surface; the use of Bresenham's algorithm to define the radial direction - because it causes an antialiasing effect (depending on the GSD of the final product); the revisits to groundels close to the ground nadir point; and false occlusion caused by multipleocclusions (for example, a building that hides another building). However, the problems with tall-narrow structures and incompatibility between image GSD and DSM resolution do not affect the results of this method.

The angle-based method also needs an interpolation step to create a gridded-DSM. However, it has some advantages when compared to the other methods, such as the ability to avoid revisits to areas close to the ground nadir point, due to its sweep method. The problems mentioned in the Z-buffer method do not apply to this method. In addition, it does not need a DBM for occlusion detection, only if a refinement is required.

The analyzed characteristics of the state-of-the-art methods can be summarized in Table I, regarding the following items:

a) the necessity of a gridded-DSM;

b) high frequency of revisit to groundels near the ground nadir point;

c) the need to use a DBM;

d) sensitive to tall narrow buildings, and to differences between image GSD and DSM resolution.

Considering these characteristics, the following section introduces the new approach for occlusion detection-SGBM together with some methodologies to generate the true orthophoto mosaics.

\section{SGBM FOR TRUE ORTHOPHOTO MOSAIC GENERATION}

Considering the drawbacks of the existing approaches, especially aiming to avoid height misrepresentation in griddedDSM, we present a new approach for occlusion detection applied to true orthophoto generation - the SGBM.

The SGBM-based true orthophoto generation has three main steps: occlusion detection by the SGBM, occlusion 


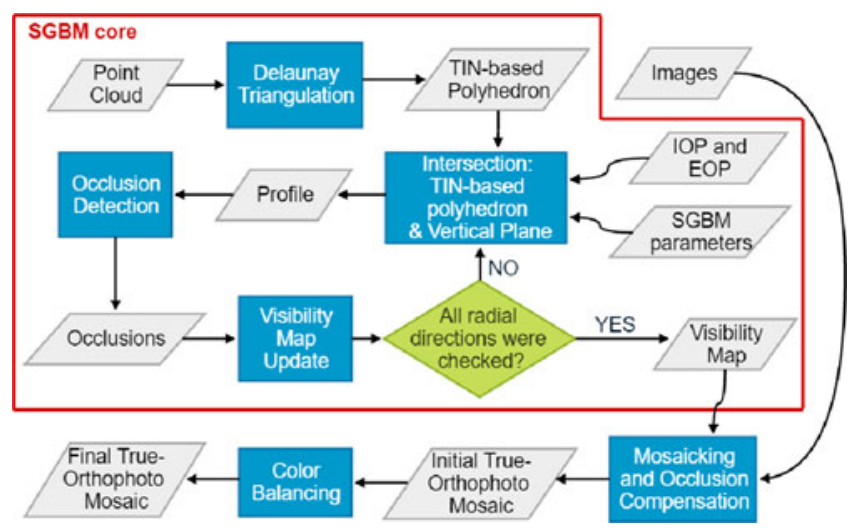

Fig. 1. SGBM-based true orthophoto method.

compensation and mosaicking, and color balancing. Fig. 1 presents an outline of the proposed method.

As a summary, the SGBM-based true orthophoto method works as follows. Initially, a point cloud is arranged following a TIN data structure [21], by applying the Delaunay constraint [22] - aiming to avoid the common drawback in the state of the art (column "a" in Table I). A vertical plane containing the PC and intercepting the TIN-based polyhedron at a selected direction defines a radial path (profile). Each profile is composed of a set of points that forms a polyline (intersection between a vertical plane that follows a radial direction and the edges of the Delaunay triangles). The analysis of the surface-gradient along the consecutive elements of this polyline allows the identification of the beginning of occluded areas in a selected radial direction. After repeating this procedure for all radial direction of all desired images, a visibility map is generated, thus finalizing the SGBM core (see Section III-A) of the proposed method. In the following (see Section III-B), the detected occlusions are compensated by using adjacent images and a cost function. This step generates a true orthophoto mosaic, which is a product free from occlusions. After the mosaic generation, a color balancing using the feathering approach (image blending - Section III-C) is applied to the previously generated mosaic, aiming at the reduction/elimination of orthoimages mosaic seamlines.

\section{A. Surface-Gradient-Based Method}

The new approach for occlusion detection proposed in this paper is described in this section. It is divided into three main steps: 1) the profile generation; 2) the surface-gradient analysis; and 3 ) the occlusion detection and visibility map generation.

1) Profile Generation: First of all, the point cloud used to represent the terrain and off-terrain objects can be obtained using a LiDAR system [23] or any image matching techniques, such as vertical line locus [24] or semiglobal matching [25].

Frame-based images are acquired in a perspective projection, which causes all occlusions to occur along radial directions from the image nadir point.

The point cloud, available in 3D, can be seen as $Z=f(X, Y)$ and organized into a TIN data structure using the Delaunay constraint, which considers that, for each triangle in a 2-D domain,

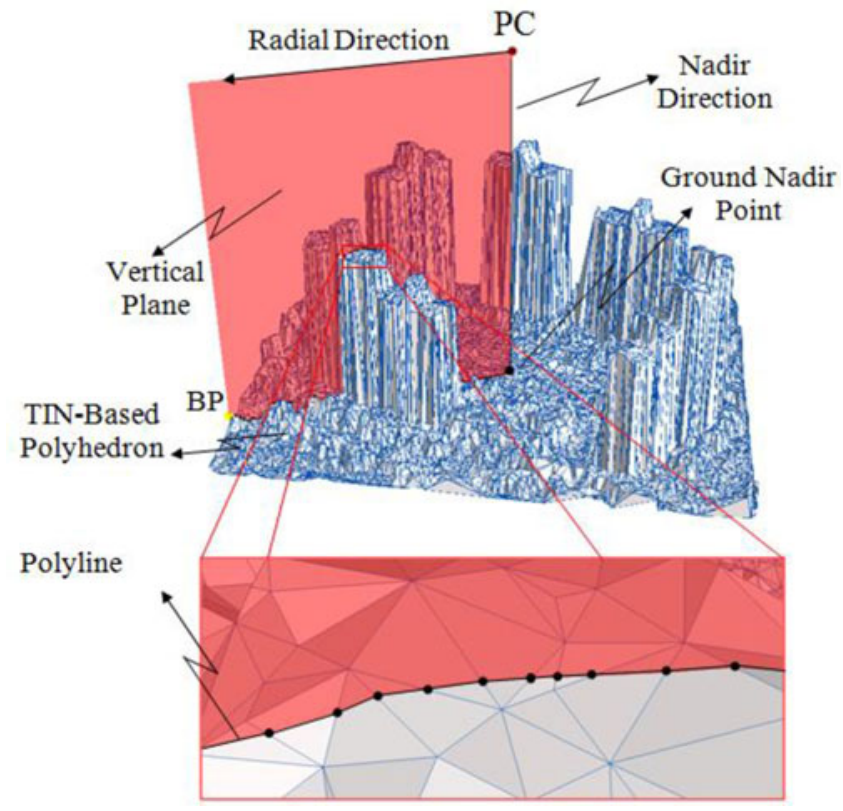

Fig. 2. Elements involved in the profile generation.

the circumference defined by their vertices must not contain any other point of the original set (see [22] and [35]). Once the TIN structure is created, the respective $\mathrm{Z}$ values are associated to each point, giving rise to a TIN-based polyhedron, where each facet corresponds to a triangle from the TIN structure.

A vertical plane containing the PC in a predefined radial direction allows the determination of a profile on the TIN-based polyhedron, as shown in Fig. 2. This radial profile defines a polyline that starts at the ground nadir point and ends at a border point (BP) of the polyhedron, which represents the terrain that underlies an image.

Fig. 2 shows that the vertical plane intersects the facets of the TIN-based polyhedron, giving rise to a polyline that is defined by a sequence of connected straight-line segments (see Fig. 3). The coordinates of each vertex of the polyline is obtained by the intersection of the vertical plane and the triangle's edges (from the TIN structure) for further use in gradient analysis, mosaicking process, and visibility map generation.

The radial direction can be defined by different ways: by angular increments or by linear increments. In this paper, the radial directions are defined by updating the coordinates of the $\mathrm{BP}$ according to one predefined parameter $\Delta \mathbf{r}$. It is important to highlight that the ground nadir point is always the beginning of a radial direction. Therefore, only the BP must be updated and its coordinates are computed in function of the step parameter $\Delta \mathbf{r}$ (see Fig. 4), which is responsible for defining how close the consecutive BPs will be from each other, and, as a consequence, the separation between consecutive radial directions. In general, the smaller $\Delta \mathrm{r}$, the more regular the building outlines in the true orthophoto, and the greater the computation effort. This parameter is numerically defined as the output image GSD and can be set by the user (if desired). At first, the image boundary projected onto the surface is determined by using Makarovic's mono plotting approach [26], represented by the corner points 


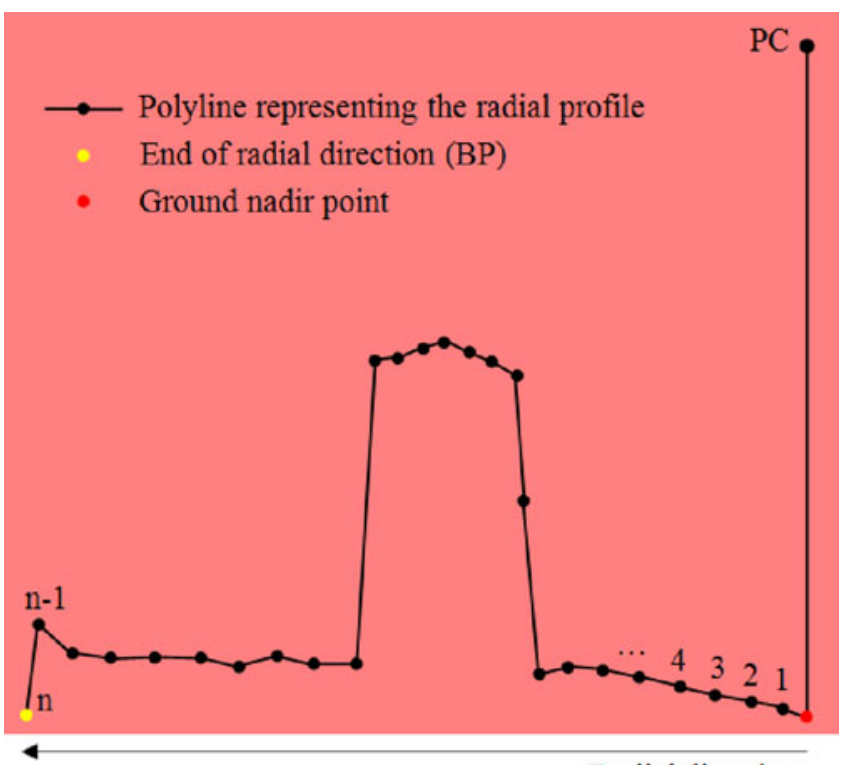

Radial direction

Fig. 3. Profile obtained from intersection between vertical plane and TINbased surface (polyhedron).

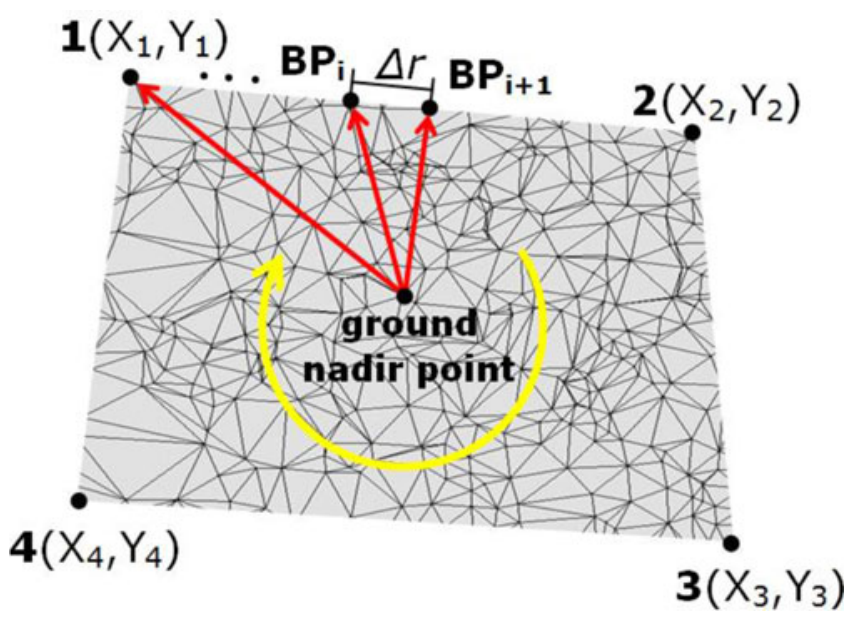

Fig. 4. Elements for radial direction definition.

TABLE II

DETERMINATION OF BP COORDINATES

\begin{tabular}{cccc}
\hline \hline Side & $\mathrm{X}$ coordinate of BP & Y coordinate of BP & BP indexing \\
\hline $1-2$ & $X_{1}+\frac{i * \Delta r}{d i s t 12} *\left(X_{2}-X_{1}\right)$ & $Y_{1}+\frac{i * \Delta r}{d i s t 12} *\left(Y_{2}-Y_{1}\right)$ & $1 \leq i<n_{12}$ \\
$2-3$ & $X_{2}+\frac{i * \Delta r}{d i s t 23} *\left(X_{3}-X_{2}\right)$ & $Y_{2}+\frac{i * \Delta r}{d i s t_{23}} *\left(Y_{3}-Y_{2}\right)$ & $1 \leq i<n_{23}$ \\
$3-4$ & $X_{3}+\frac{i * \Delta r}{\text { dist }_{34}} *\left(X_{4}-X_{3}\right)$ & $Y_{3}+\frac{i * \Delta r}{\text { dist }_{34}} *\left(Y_{4}-Y_{3}\right)$ & $1 \leq i<n_{34}$ \\
$4-1$ & $X_{4}+\frac{i * \Delta r}{\text { dist }_{41}} *\left(X_{1}-X_{4}\right)$ & $Y_{4}+\frac{i * \Delta r}{\text { dist }_{41}} *\left(Y_{1}-Y_{4}\right)$ & $1 \leq i<n_{41}$ \\
\hline \hline
\end{tabular}

1, 2, 3, and 4 in Fig. 4. The first BP is taken at corner point 1 and is updated, in clockwise direction, considering the $\Delta \mathbf{r}$ value, until it reaches corner point 1 again. The coordinates of each BP are updated by the formulation presented in Table II.

Basically, the BP coordinates are updated $\mathrm{n}_{\mathrm{kj}}$ times for each side of the polyhedron that underlies the current image (defined by two vertices $\mathrm{k}$ and $\mathrm{j}$, that change for each side $\left[\mathrm{n}_{12}, \mathrm{n}_{23}\right.$, $\left.\mathrm{n}_{34}, \mathrm{n}_{41}\right]$ ) - see Fig. 4. The computation of $\mathrm{n}_{\mathrm{kj}}$ is as follows: $\operatorname{int}\left(\operatorname{dist}_{\mathrm{kj}} / \boldsymbol{\Delta r}\right)$, where int stands for integer of a real number and dist $_{\mathrm{kj}}$ is the distance between the vertices $\mathrm{k}$ and $\mathrm{j}$. This formulation will allow the determination of the necessary number of radial directions (between $\mathrm{k}$ and $\mathrm{j}$ ) to properly identify the occlusion areas.

Since all radial directions are defined, it is possible to analyze the surface-gradients for each radial profile.

2) Surface-Gradient Analysis: The surface-gradient analysis is the key-step for applying the SGBM. Considering Fig. 3, for a given radial direction starting at the ground nadir point, each straight-line segment of this radial polyline is defined by two 3-D endpoints. The coordinates of two consecutive endpoints allow the estimation of surface gradient $\left(\frac{\partial Z}{\partial r}\right)$, which can be considered as the slope of each straight-line segment, by the following:

$$
\text { If } 1 \leq i \leq n_{p}-1, \quad \frac{\partial Z}{\partial r}=\frac{Z_{i+1}-Z_{i}}{r_{i+1}-r_{i}}
$$

where $\mathrm{n}_{p}$ is the number of points that defines the current profile, and $\mathrm{r}_{i}$ can be defined as: $r_{i}=i$, i.e., it is the vertex index on a sequence of points that defines the current radial profile; or the horizontal distance between two consecutive vertices ( $i$ and $i+1)$ of the current radial profile.

We opted for the first definition $\left(r_{i}=i\right)$ because of the following.

1) As $r_{(i+1)}-r_{i}=1$, the gradient computed using this definition is in metric unit and gives the local surface variation $\left(Z_{(i+1)}-Z_{i}\right)$.

2) As the gradient denominator for the second definition is always positive (assuming that $r_{(i+1)} \neq r_{i}$ ), it is only necessary to check whether the height difference $\left(Z_{(i+1)}-Z_{i}\right)$ is negative or not.

3) Most important, the gradient in terms of height difference $\left(Z_{(i+1)}-Z_{i}\right)$ is much more intuitive for setting up a threshold that allows the elimination of spurious or insignificant occlusions (if desired).

A negative surface gradient indicates the start point of an occlusion area. However, to minimize the influence of noise, a threshold (Th) can be applied. This threshold is defined as the height of the shortest object that can cause a preeminent occlusion for a specific application.

So, if the condition $\frac{\partial Z}{\partial r} \leq-\boldsymbol{T} \boldsymbol{h}$ is satisfied, the start point of an occlusion is found. After detecting this position, it is possible to project it onto the TIN-based polyhedron and to identify the extent of the occlusion. Usually, point-based approaches were used for verifying obstructions along radial directions. However, in the following section, we introduce the concept of occluded triangles, which are further used for constructing the visibility map.

3) Occlusion Detection and Visibility Map: The occlusion detection is accomplished by projecting the point that represents the start of a negative surface-gradient (points $\mathrm{A}$ and $\mathrm{C}$ on the aboveground objects-see Fig. 5) onto the TIN-based polyhedron, being possible to identify points $\mathrm{B}$ and $\mathrm{D}$ (end of the occlusions caused by the buildings), respectively. This projection is established by the identification of the polyhedron 


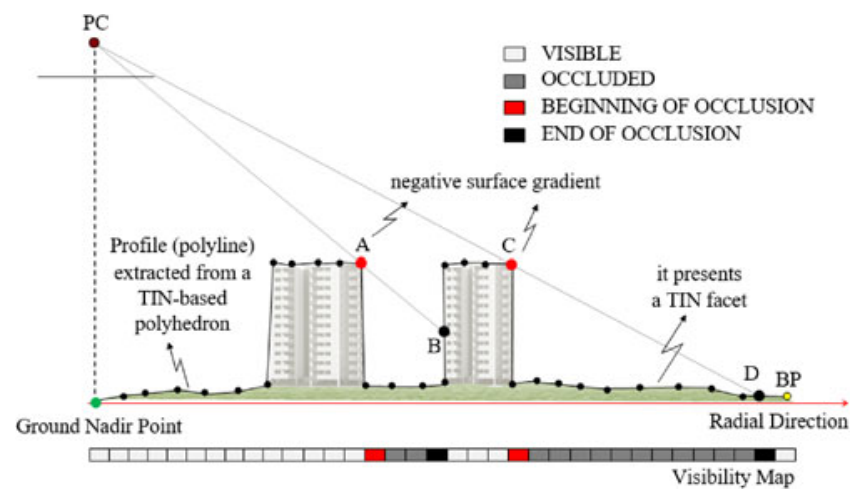

Fig. 5. Determination of the end of an occlusion and the filling of a visibility map.

triangles $\left(T_{s}\right)$ that is intersected by the lines passing through the beginning of occlusions and the PC. The intersections (points $\mathrm{B}$ and $\mathrm{D}$ in Fig. 5) determine the triangles that correspond to the end of the occlusions. This idea brings up the concept of an occluded triangle, instead of an occluded groundel. However, the triangle might be partly visible and partly occluded.

For the building closest to the nadir direction (see Fig. 5), the occlusion area is a set of adjacent facets (triangles) that starts from the triangle containing point $\mathrm{A}$ and ends at triangle $T$-that contains point B. This set of triangles is then stored in the visibility map-with a proper projection of the groundels enclosed in the triangles, and also those that cross the triangle's edges, onto the visibility map. The visibility map is a matrix with the same spatial resolution and dimension as the output image, which can be the true orthophoto mosaic in a situation where several images are used. The visibility map will contain the information of visibility for every single pixel in all images, allowing the true orthophoto mosaic algorithm to identify the image(s) that a given true orthophoto pixel is visible.

At this point, two considerations must be done: 1) As the intersected triangle $T$ is considered completely occluded, it may extrapolate the real occlusion extension. However, due to the high point cloud density this extrapolation is not a problem since these small-extrapolated areas are compensated by radiometric information from adjacent images, in a further step; 2) The set of triangles, stored as occlusion area, are not revisited, even if the adjacent radial direction is close to the current one (in the case of a small value for $\Delta \mathbf{r}$ ), which provides a shorter processing time.

The search for the triangle $T$ along the radial direction is accomplished only for the polyline segments after point A, sequentially. In case of multiple occlusions - object(s) hiding other object(s) - the method is able to identify the ends of the occlusion on a triangle that represents a wall, for example - this is a problem found in some methods in the state of the art (common situation in high dense urban areas) - see Fig. 5.

As the images are acquired with end lap and side lap, a specific area on the ground can be occluded in some images and visible in others. The visibility map allows the identification of the image that is the best source of information to compensate the occlusion and to create the true orthophoto mosaic.

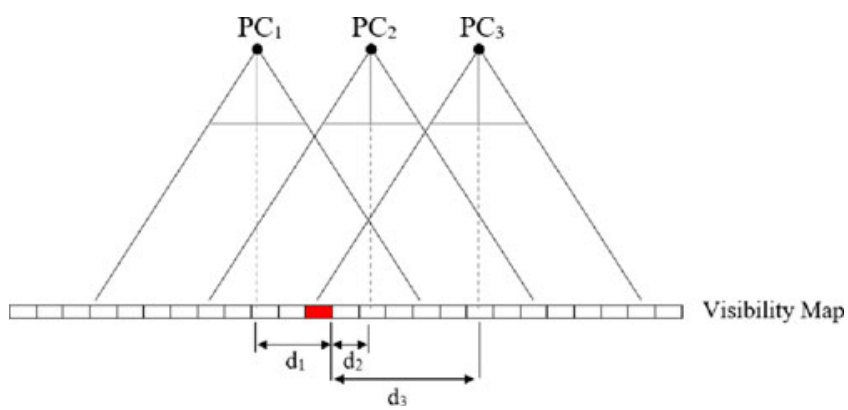

Fig. 6. Example of cost function - nearest ground nadir method.

\section{B. Occlusion Compensation and Mosaicking}

Usually, in photogrammetric projects several images are acquired and, as a consequence, a mosaic of all true orthophotos must be created to cover all the interest area. The mosaicking is not a simple process and requires several specific procedures.

In the case of generating true orthophotos, the mosaicking process requires a cost function to determine which image must be the source of radiometric/spectral information. After the best image is identified, considering a cost-function and the available dataset, the visibility map is used to check whether the pixel marked as occluded is visible in the selected image. If it is identified as nonvisible, other images are checked until a valid visible pixel is found, following the priority order dictated by the cost-function.

In this paper, two cost-functions are compared: the nearest ground nadir method and the minimum angle method, as introduced by [27]. Also, the color balancing through a feathering approach is applied and analyzed.

1) Nearest Ground Nadir Method: The most common costfunction used by aerial image mosaicking approaches considers the proximity between ground nadir points of the images and a given groundel, aiming to define, e.g., which image is the best source of radiometric information to fill an occlusion. The further a pixel is from the image nadir, the more severe the relief displacement is [2]. Therefore, the image that has the nearest ground nadir point to a given occlusion is potentially considered the best source of radiometric information to fill an occlusion and to create the mosaic.

This approach selects the best image by checking the distance between the current analyzed groundel and the ground nadir point of the image, for every single image. In the example presented in Fig. 6, the highlighted groundel (in red) is visible in all three images. Nevertheless, the closest ground nadir point to this groundel is related to $\mathrm{PC}_{2}$ whose distance $d_{2}$ is the smallest. Therefore, image 2 is defined as the best source for radiometric information. Additional information about the nearest ground nadir approach can be found in [3].

Larger image end lap and side lap make possible the use of central areas of several images, minimizing these occlusions. However, the increase of end and side laps does not guarantee the absence of occlusions; in fact, this alternative will only mitigate the effect and require a longer processing time and more flight lines. 


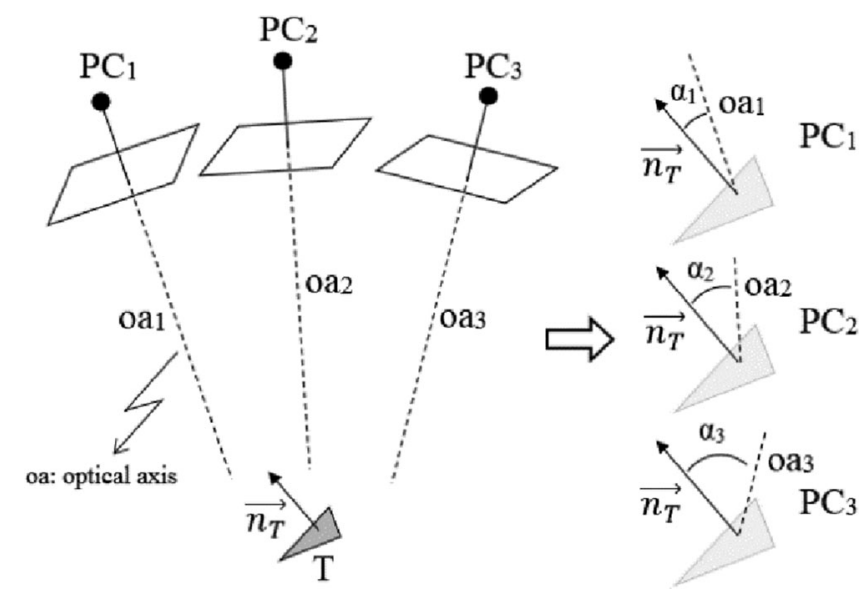

Fig. 7. Example of cost function-minimum angle method.

2) Minimum Angle Method: This method checks the orientation between the line of sight of a polyhedron facet and the optical axis of the camera at different exposure times, aiming to find the image acquired with the optical axis that was as nearly parallel to the normal vector of the tested polyhedron facet as possible. In this paper, this strategy is denominated as the minimum angle method. The angle formed by the camera optical axis and the facet line of sight vary between adjacent images (end lap or side lap), and this variation is more critical in situations where only nonvertical images are used, such as heritage structure mapping, and when large segmented areas are utilized, instead of small triangles, such as roof planes, as presented in, e.g., [28]. The work presented by [12] also uses a similar approach to define an ideal image selection.

The image that corresponds to the minimum angle is the source to fill up the occlusion with proper radiometric information.

Fig. 7 presents an example with three different images. The angles $\left(\alpha_{1}, \alpha_{2}, \alpha_{3}\right)$ between a triangle's normal vector $\left(\overrightarrow{n_{T}}\right)$ and the camera's optical axes $\left(\mathrm{oa}_{1}, \mathrm{oa}_{2}, \mathrm{oa}_{3}\right)$, respectively, are shown. In this case, the angle $\alpha_{1}$ is the smallest, which makes image 1 the optimal radiometric source to compensate for the occlusion for triangle $T$, even if $\mathrm{PC}_{2}$ corresponds to the image with the nearest ground nadir point to $T$.

\section{Color Balancing}

As the images are taken at different exposure times for a single flight mission, they can be collected with different external influences, such as the sun light intensity. Also, correspondent areas can appear with different luminosity due to the vignette effect and the change in the viewpoint. This variation of brightness results in radiometric dissimilarity and discontinuity in the true orthophoto mosaic, which should be compensated for. The transition between two adjacent images is known as seamline (color transition).

In a conventional orthorectification process (based on a DTM), it is important to identify and manipulate the seamlines, aiming to avoid variation in the representation of objects in two different perspective views (a building for example). Therefore,

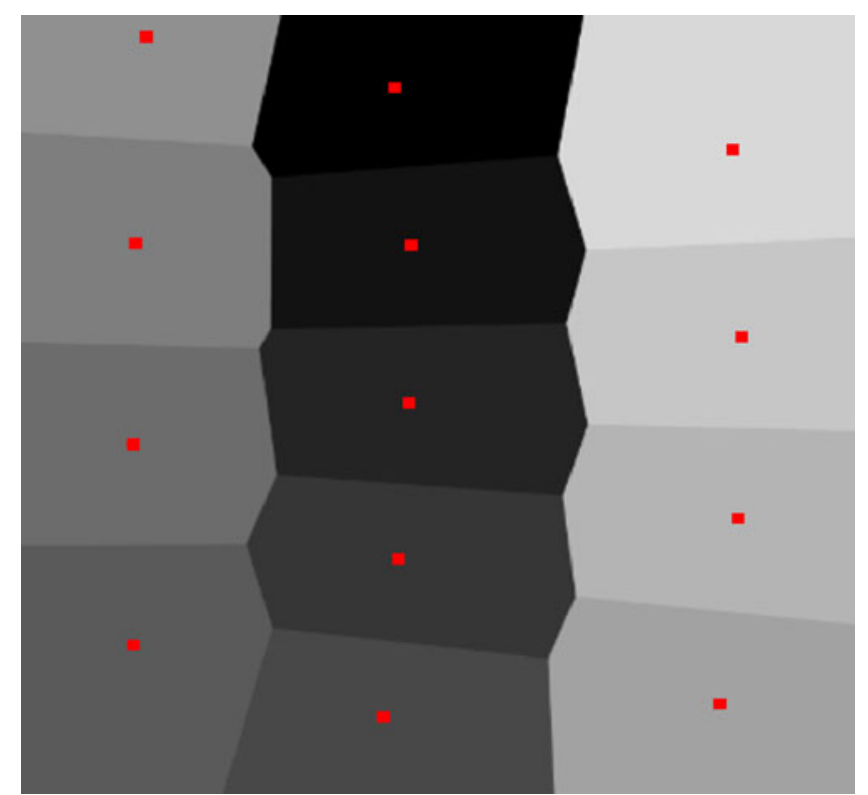

Fig. 8. Voronoi diagram from the image block.

the seamlines should not cross any elements with height variation, due to the different radial displacements in adjacent orthophotos. This concern is not an issue in true-othophoto mosaic generation, because all elements above the ground are projected in their orthogonal location, i.e., adjacent images do not have misalignment of features, assuming that an image block with high-quality exterior and interior orientation parameters-EOPs and IOPs, respectively - as well as an accurate DSM are available. Consequently, for true orthophoto mosaic generation, only the color variation adjustment, or balancing, needs to be addressed.

In order to perform the color balancing, it is necessary, first, to identify the seamlines on the true orthophoto mosaic. Considering the ground nadir point of each image of the block as one seed, the seamlines can be automatically determined by using the Voronoi diagram generated by this structure. The Voronoi diagram, which is a dual representation of the Delaunay triangulation, expresses the same geometric constraints obtained by the nearest ground nadir approach, due to the division of a plane in regions based on the distance between the input set of points, i.e., the ground nadir points.

Fig. 8 presents a Voronoi diagram obtained by using $13 \mathrm{im}-$ ages. The Voronoi cells in the diagram are represented by different colors (gray scale), and the original input points (ground nadir points) are represented by red dots.

The generation of a Voronoi diagram allows not only the choice for the best image (according to the first cost-function) but also the automatic recovery of cell edges, which corresponds to the seamline locations. Since each seamline is identified, it is possible to apply a feathering (image blending) to minimize the radiometric differences near the transition region, and then generate a true orthophoto mosaic free from visible seamlines, or at least minimize its effect.

The feathering approach aims to eliminate the seamlines between adjacent images by applying a weighted average method 

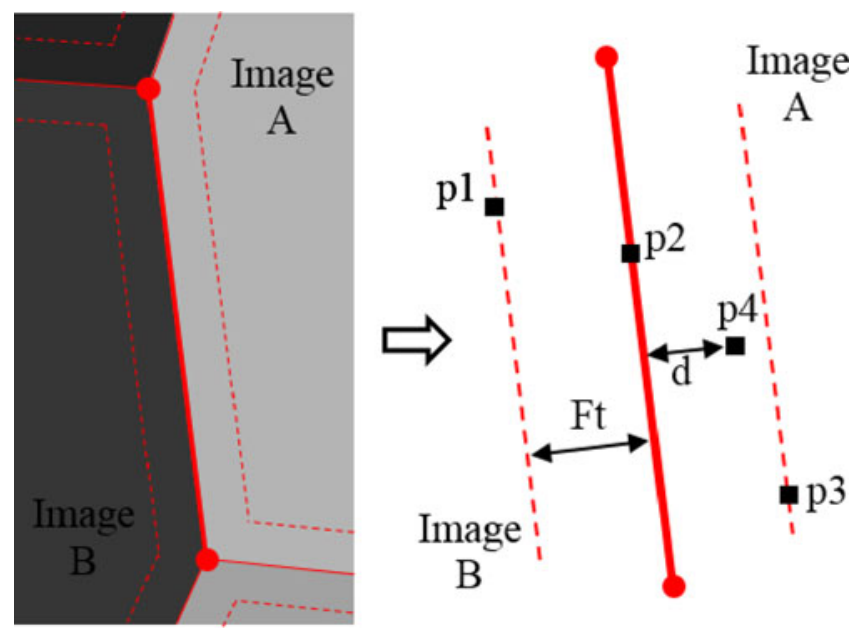

Fig. 9. Image feathering.

TABLE III

PERCENTAGE OF RADIOMETRIC INFORMATION FROM EACH IMAGE IN THE FEATHERING METHOD (SEE FIG. 9)

\begin{tabular}{lcc}
\hline \hline Pixel & $\%$ from image A & $\%$ from image B \\
\hline p1 & 0 & 100 \\
p2 & 50 & 50 \\
p3 & 100 & 0 \\
p4 & {$\left[\frac{50 * d}{F t}+50\right]$} & $100-\left[\frac{50 * d}{F t}+50\right]$ \\
\hline \hline
\end{tabular}

[29]. Fig. 9 presents all necessary elements for understanding the image feathering. The seamline between images A and B is represented by a thick red line. To apply the feathering, it is necessary to define a buffer width $\mathbf{F t}$ (half-width of the red dashed buffer in Fig. 9) that defines the region where the pixels will be combined. The final true orthophoto mosaic pixels (output) are obtained by a linear combination between images A and B, only if the pixel is located inside the buffer. The percentage of intensity contribution from each image is determined based on the distance " $\mathrm{d}$ " between the correspondent groundel and the seamline. If the distance is equal or larger than $\mathbf{F t}$, the output pixel is $100 \%$ of its correspondent Voronoi cell. If the distance is zero, the output pixel uses $50 \%$ of both Voronoi cells, i.e., the output digital number will be the average of both images. For a general case, the output radiometric information will be a linear combination of those pixels in both images, according to the equation shown in Table III for pixel p4. For the example presented in Fig. 9, the percentage values for each pixel $(p)$ are listed in Table III.

\section{EXPERIMENTS AND QUALITY ASSESSMENT}

This section shows experiments performed using two datasets. The following sections present: the dataset description; a comparison between four occlusion detection methods applied to the same dataset; an evaluation of occlusion detection completeness and time of processing; a comparison between cost-functions presented in Section III-B; the verification of alignment quality on final product; two true-othophoto mosaics

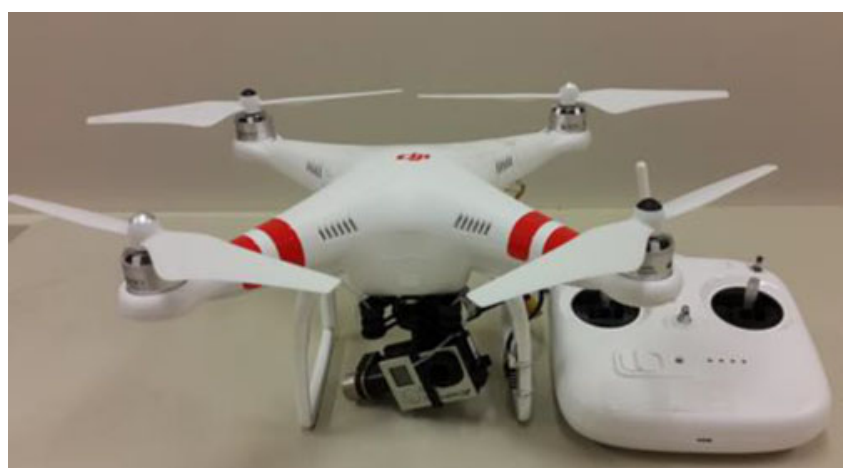

Fig. 10. DJI Phantom $2+$ GoPro camera.

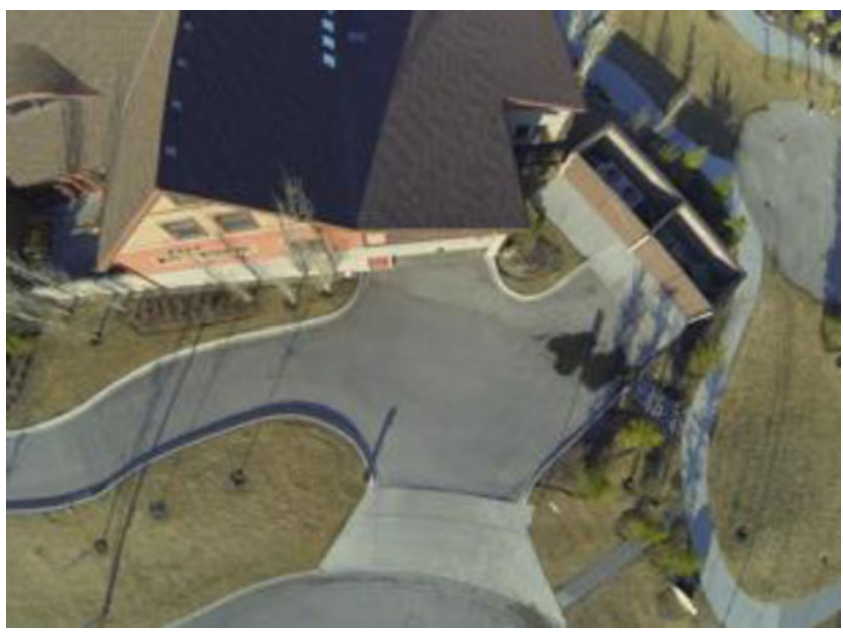

Fig. 11. Example of image from dataset 1.

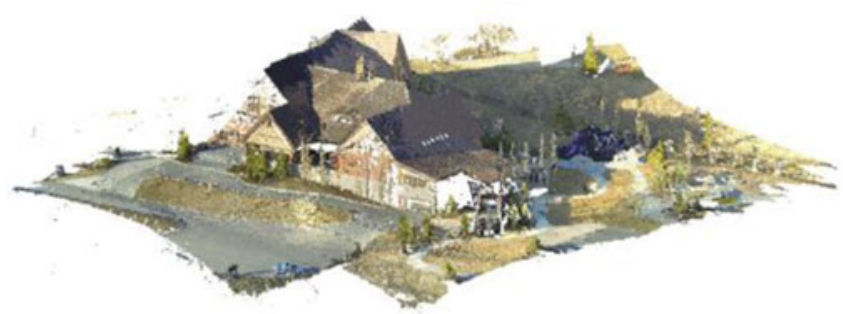

Fig. 12. Example of point cloud from dataset 1.

generated by using the SGBM for occlusion detection; and the advantages and disadvantages of the proposed method.

\section{A. Dataset Description}

1) Dataset 1: Dataset 1 was collected by a low-cost unmanned aerial vehicle (UAV) platform at an average flying height of $25 \mathrm{~m}$. The platform is a DJI Phantom 2 attached with a gimbal and a GoPro camera HERO $3+$ (see Fig. 10). A total of 81 images were acquired (example in Fig. 11) with a GSD of around $3 \mathrm{~cm}$. The point cloud (see Fig. 12) was photogrammetrically derived by using an image matching technique that improves the result obtained by the semiglobal image matching, as presented in [30], and has an average point density of 100 points $/ \mathrm{m}^{2}$. 


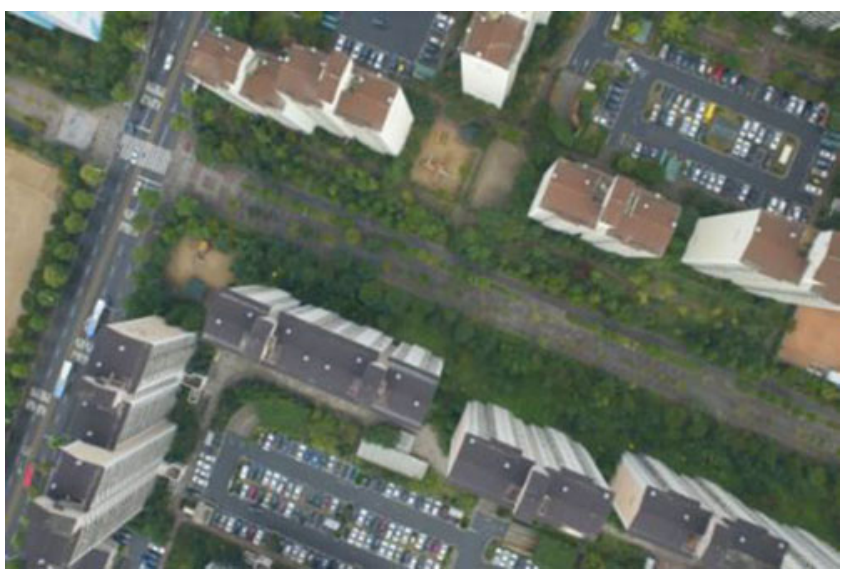

Fig. 13. Example of image from dataset 2 .

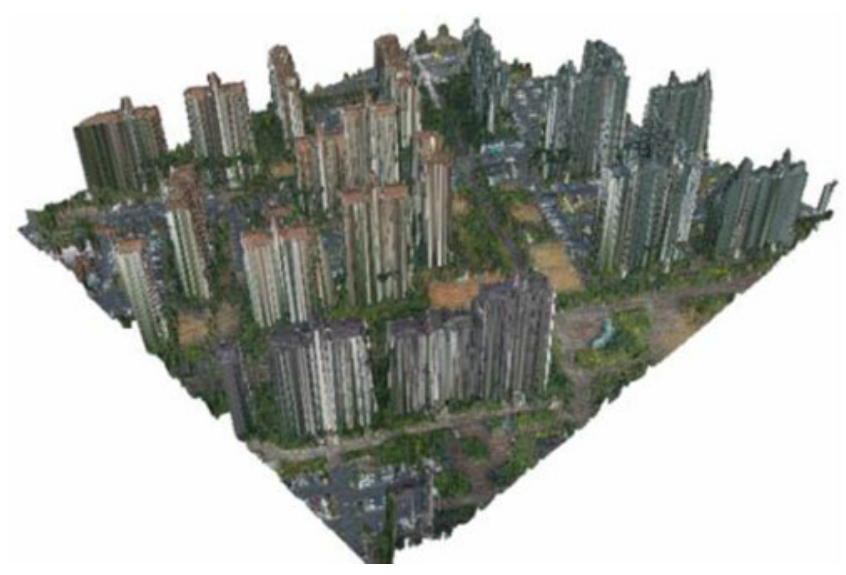

Fig. 14. Example of point cloud from dataset 2.

2) Dataset 2: This dataset was acquired by a conventional aerial mission, i.e., by using a manned aircraft. It is composed of 13 images taken by a Canon EOS-1D camera with an average flying height of $240 \mathrm{~m}$. The average GSD is around $9 \mathrm{~cm}$ (example in Fig. 13). The point cloud was obtained by a LiDAR system $\left(8\right.$ points $\left./ \mathrm{m}^{2}\right)$ and can be seen, as a triangulated surface (color-coded), in Fig. 14.

These datasets were selected because of the relative low flying heights, which cause the presence of larger occlusion areas.

\section{B. Quality Assessment Approach}

The quality assessment was accomplished by using the occlusion detection and the final true orthophoto mosaic as input information. The occlusion areas detected by the SGBM are evaluated in terms of completeness. In the final product, the true orthophoto mosaic, the seamline mismatches are also analyzed.

Completeness is a quality index that evaluates the percentage of a reference polygon (extracted manually in this paper) detected as an occlusion area by the SGBM. Analysis of the completeness allows the evaluation of the reliability of the proposed method regarding occlusion detection. Similar approaches can be seen in [31] and [32].

Next (see Fig. 15), the alignment of linear features on the final product is evaluated by checking the distance between a straight

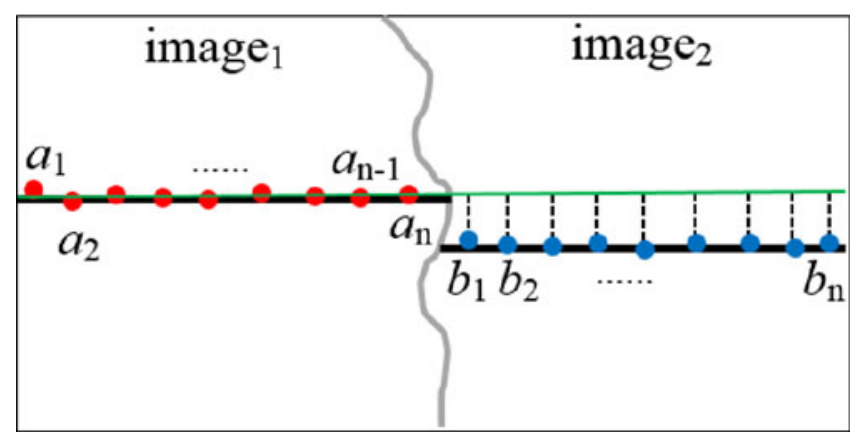

Fig. 15. Approach for seamlines mismatch evaluation.

line (green), derived from points representing a linear feature in one image $\left(a_{1}, a_{2}, \ldots, a_{n}\right)$, and the points in the second image that represent the same linear feature $\left(b_{1}, b_{2}, \ldots, b_{n}\right)$. The root-mean-square error (RMSE) of these distances can be defined as the alignment error. The alignment analysis is important to evaluate the quality of the block triangulation (IOPs and EOPs) and the quality of the DSM.

\section{Comparison Between Occlusion Detection Methods}

Considering the importance of completeness regarding occlusion detection, this experiment shows a qualitative comparison of four different methods of occlusion detection. Fig. 16 contains the results using dataset 2 by applying: 1) Z-buffer; 2) anglebased with spiral sweep method; 3 ) angle-based with adaptive radial sweep method; and 4) SGBM.

The result obtained by the Z-buffer method is visually less complete when compared to the others, due to the false visibilities. On the other hand, both Angle-based and SGBM show more complete occlusion detection. One aspect that can be observed is the reduced number of regions labeled as occlusion in some streets and parking lots in Fig. 16(d). These false occlusion detections [see Fig. 16(a), (b), and (c)] occur due to interpolation errors in the gridded-DSM representation, which is not a problem when applying a TIN data structure, such as in the SGBM results [see Fig. 16(d)]-which show a proper occlusion detection.

\section{Occlusion Completeness Evaluation}

Completeness is an important quality index for occlusion detection evaluation, because if an occlusion is not completely identified, i.e., part of it is considered as visible, the true orthophoto mosaic will have remaining wrong representation of the objects above ground. Regarding this statement, the occlusion detection of two areas from dataset 2 was assessed.

The reference polygon (red polygon in Figs. 17 and 18) was extracted manually from the double-mapped representation, where it is possible to define exactly the beginning and end of occlusions.

The completeness values (see Table IV) determined for each method indicates an advantage of SGBM in relation to Z-buffer and Angle-based methods. The Z-buffer method has incomplete occlusion detection and should not be applied to areas with 

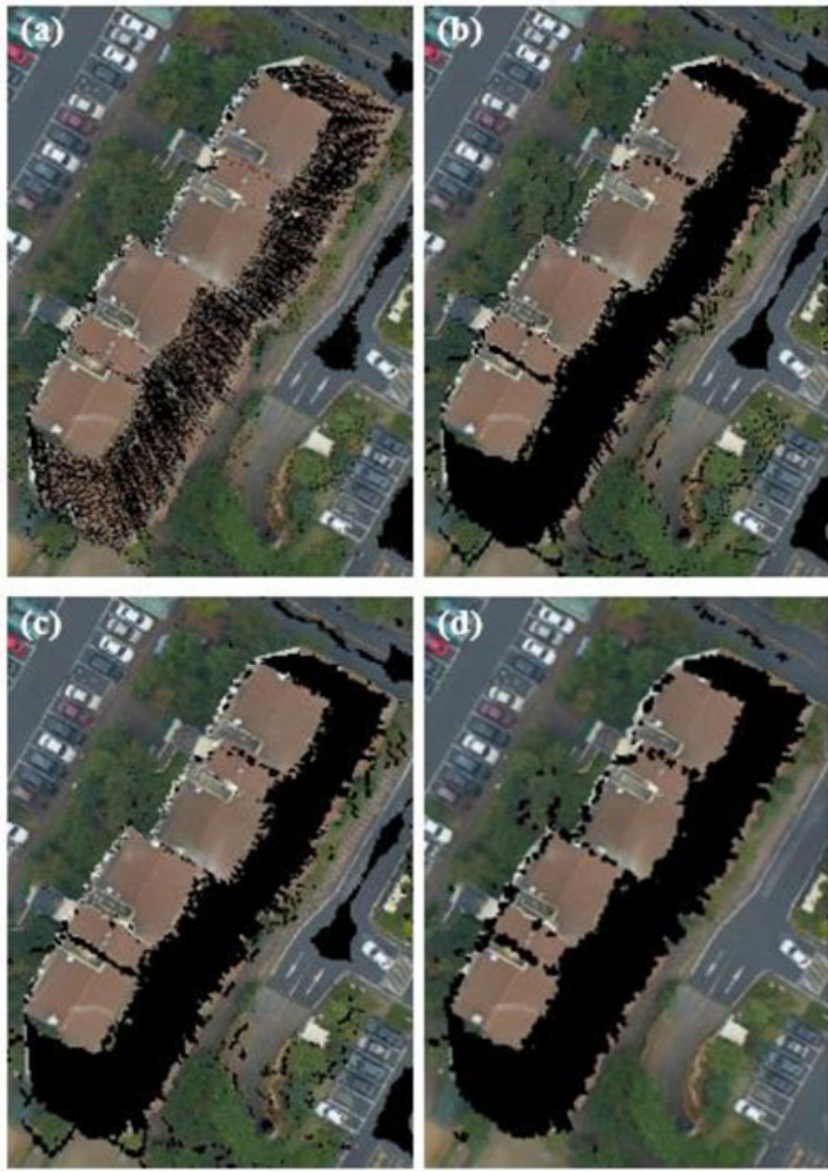

Fig. 16. Occlusion detection by applying: (a) Z-buffer; (b) Angle-based with spiral sweep method; (c) Angle-based with adaptive radial sweep method; (d) The proposed method-SGBM.

dense urban characteristics due to the problems with tall narrow buildings and noncompatibility between the nominal GSD of the image and the DSM resolution. The false visibilities can be minimized by using morphological operators as shown in [20] or by adding pseudogroundels, as discussed in [4].

Based on the completeness values, for both experiments, it is possible to see that the Z-buffer method gives lower completeness, followed by Angle-based (spiral and radial, respectively). Considering the Angle-based (radial) results, the completeness of SGBM was $2.4 \%$ and $4.8 \%$ higher for experiments 1 and 2 , respectively.

The time of processing for each method, presented in Table IV, applied to the same image and DSM, is shown in Table V. The specifications of the used computer are the following: Processor Intel CoreTM i7-4500U 2.4 GHz, RAM of 8 GB and hard drive with 1 TB.

It is important to highlight that these values do not include the time for generating a gridded-DSM (essential for the first three methods - Z-buffer and Angle-based). Even not considering the interpolation step, it is possible to see the advantage of the SGBM when compared to the other state-of-the-art methods. The main reason for this performance is the fact that the SGBM identify occluded triangles instead occluded DSM cells, which

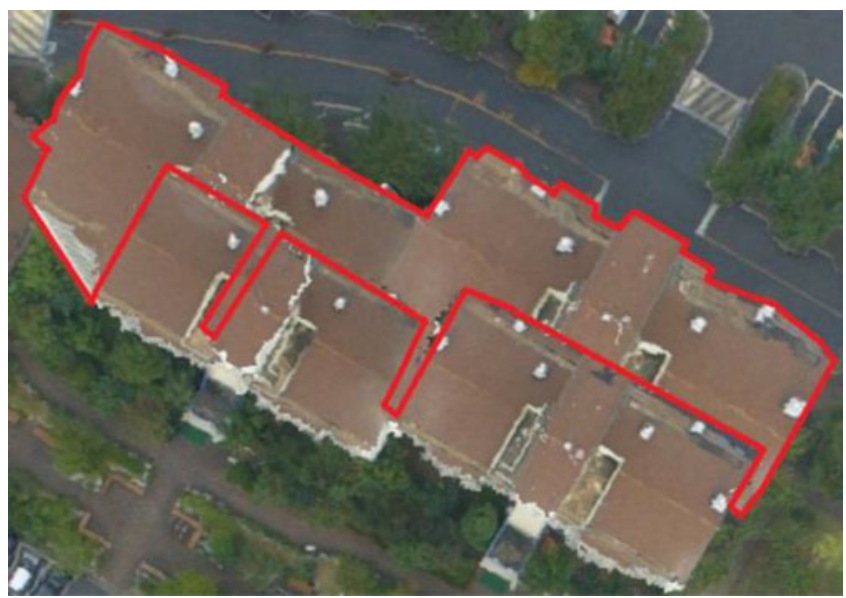

Fig. 17. Area of experiment 1 that contains the double mapping, together with the reference polygon for completeness evaluation.

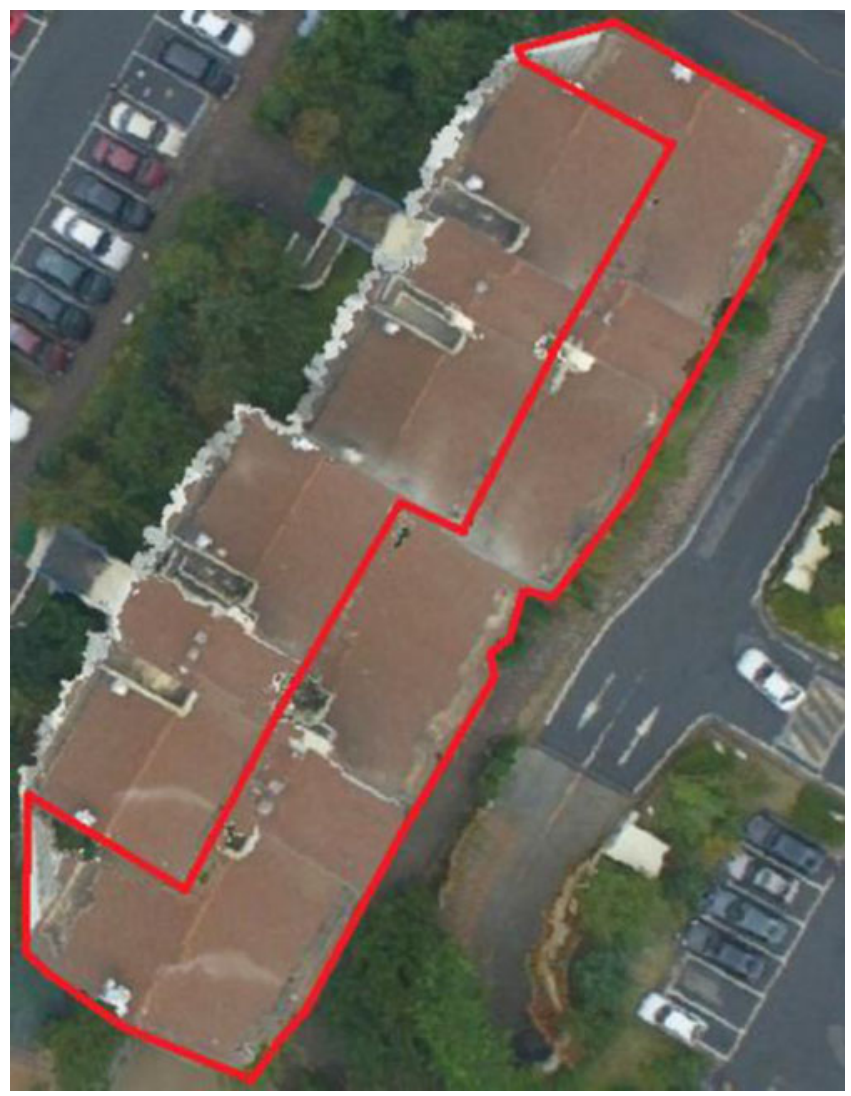

Fig. 18. Area of experiment 2 that contains the double mapping, together with the reference polygon for completeness evaluation.

reduce the processing time during the occlusion identification, because the pixels (in the visibility map) that compose a triangle are already labeled as occluded. As a result, it is not necessary to check them in the next radial direction (profile).

\section{E. Sensitivity Analysis of Th Parameter}

As described in Section III, the SGBM uses the so-called Th parameter to minimize the presence of noise on the occlusion 
TABLE IV

COMPLETENESS VALUES OF THE EXPERIMENTS

\begin{tabular}{lcc}
\hline \hline Methods & \multicolumn{2}{l}{ Completeness (\%) } \\
\cline { 2 - 3 } & Area 1 & Area 2 \\
\hline Z-buffer & 45.30 & 53.98 \\
Angle-based: spiral & 93.28 & 83.99 \\
Angle-based: radial & 94.10 & 87.00 \\
SGBM & 96.54 & 91.80 \\
\hline \hline
\end{tabular}

TABLE V

TIME OF PROCESSING FOR OCCLUSION DETECTION

\begin{tabular}{lcc}
\hline \hline \multirow{2}{*}{ Methods } & \multicolumn{2}{c}{ Time of processing (min) } \\
\cline { 2 - 3 } & Area 1 & Area 2 \\
\hline Z-buffer & 2.57 & 2.50 \\
Angle-based: spiral & 1.00 & 1.01 \\
Angle-based: radial & 1.15 & 1.08 \\
SGBM & 1.15 & 0.90 \\
\hline \hline
\end{tabular}
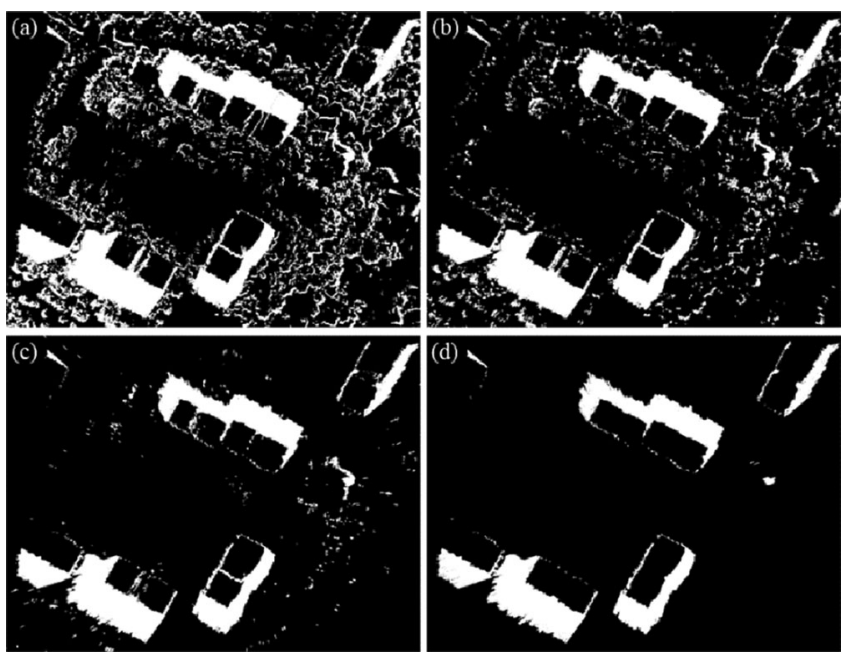

Fig. 19. Visibility maps showing the sensitivity of occlusion detection by changing parameter Th: (a) $1 \mathrm{~m}$; (b) $3 \mathrm{~m}$; (c) $5 \mathrm{~m}$; and (d) $10 \mathrm{~m}$.

detection. This threshold can define the minimum object height to be considered on the occlusion identification, by establishing a restriction during the surface gradient analysis. The values set for this parameter can have an influence on the results obtained. Fig. 19 presents the use of different thresholds for an area enclosed by dataset 2 .

For this experiment, only objects higher than $1,3,5$, and 10 m, presented in Fig. 19(a), (b), (c), and (d), respectively, was considered.

As can be seen in Fig. 19, the parameter Th has great influence on the quantity of occluded objects detected. Bearing in mind that the significant occlusion presence on aerial images over urban areas comes from tall buildings, the possibility of setting this threshold can avoid unnecessary processing, which decreases noise on the results, as well as the processing time.
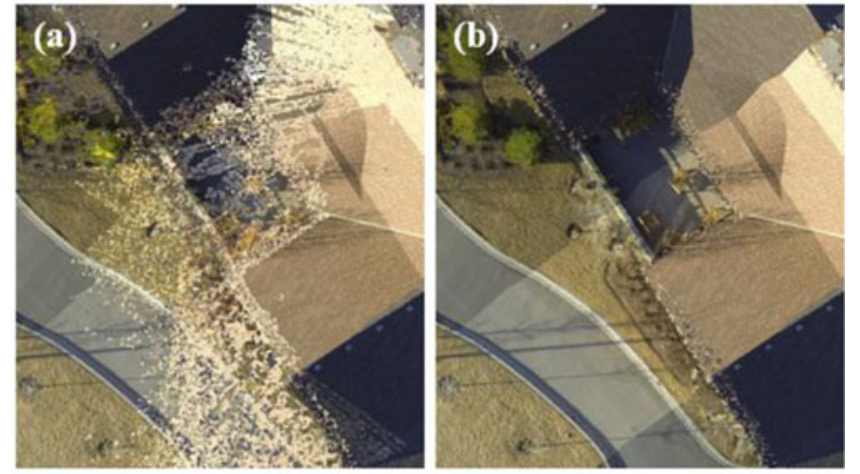

Fig. 20. Mosaicking using the cost-function: (a) Minimum angle method; (b) Nearest ground nadir method.

\section{F. Comparison of Cost-Functions for the Mosaicking Process}

As presented in the previous section, the mosaicking process uses a cost-function to define which image is most suitable for occlusion compensation and image mosaicking, when multiple overlap images are available. The following experiment presents different results obtained by applying the minimum angle method [see Fig. 20(a)] and the nearest ground nadir method [see Fig. 20(b)]. This area is composed by several images such as the one presented in Fig. 11.

Considering point clouds with high density (triangles with small areas), small variations at height coordinates of the vertices (noise) generate very heterogeneous directions for the normal vectors. Therefore, neighboring triangles can be pointing to different directions, even for a flat and homogeneous area. As a result, the best image to compensate for an occlusion using the minimum angle method can produce unexpected results. This is the reason for the "noise" found in Fig. 20(a). Considering these results, the SGBM uses the nearest ground nadir approach to generate the true orthophoto mosaics [see Fig. 20(b)].

It is important to highlight that if the triangles, or any other planar feature, have a larger dimension, the approach that takes into account the surface orientation is useful and should be considered, as can be seen in [28].

\section{G. Assessment of Mismatch in True Orthoimagery Mosaic}

The evaluation of the alignments in true orthophoto mosaic was performed using dataset 1 , because of the better spatial resolution and presence of linear features, which allow an easy identification of seamlines. It is important to highlight that the alignment depends on the quality of the IOPs, the EOPs (from the bundle block adjustment or direct georeferencing system), and the surface representation (point cloud accuracy). This experiments aim to show the consistent alignment along linear features after the true orthophoto mosaic generation.

Fig. 21 presents the test areas used in this evaluation. The first test area shows a ridge of a roof (green line) on the building that appears in Fig. 11, that crosses two adjacent images [see Fig. 21(a)]. The second test area shows a fence at ground level [see Fig. 21(b)-green line]. For a true orthophoto mosaic, the linear features (such as the roof ridge and the fence) must be well 

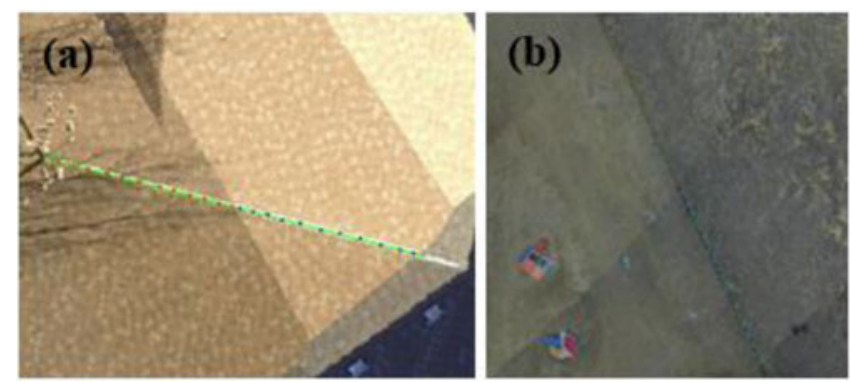

Fig. 21. Alignment evaluation. (a) Roof ridge and (b) Ground level.

TABLE VI

ERRORS ON THE LINEAR FEATURES ALIGNMENT

\begin{tabular}{lccc}
\hline \hline Area & RMSE (pixel) & GSD (m) & RMSE (m) \\
\hline 1 & 0.10 & 0.03 & 0.003 \\
2 & 0.14 & 0.03 & 0.004 \\
\hline \hline
\end{tabular}
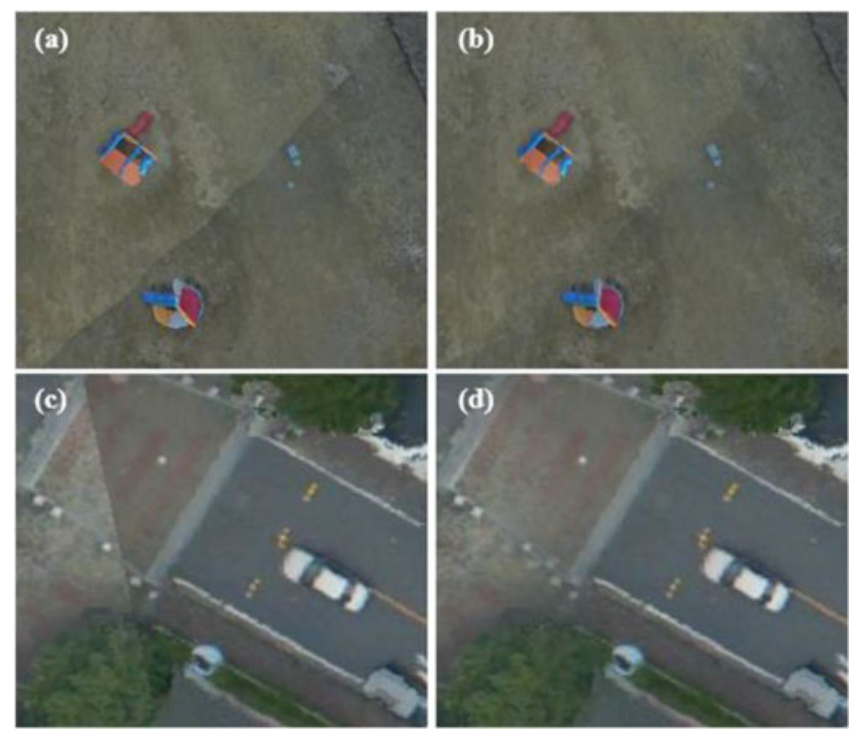

Fig. 22. Color balancing results considering $\mathrm{Ft}=10$ pixels. (a and $\mathrm{c}$ ) Before. (b and d) After.

aligned. The misalignments in terms of RMSE of the distances (see Fig. 15) are shown in Table VI. The mismatch errors are at the subpixel level for both datasets.

The points that represent the lines were manually identified immediately before the color balancing procedure, when it is easier to identify the linear features located across seamlines. After the color balancing, the seamlines are not visible [as can be seen in Fig. 22(b) and (d)]. These results show a good alignment of adjacent imagens in both dataset and a proper color compensation to mitigate the seamlines presence.

\section{H. True Orthophoto Mosaics}

In this section, the true orthophoto mosaics, obtained from datasets 2 and 1 with color balancing, are shown in Figs. 23 and 24 , respectively. The results from dataset 1 have a GSD of $3 \mathrm{~cm}$

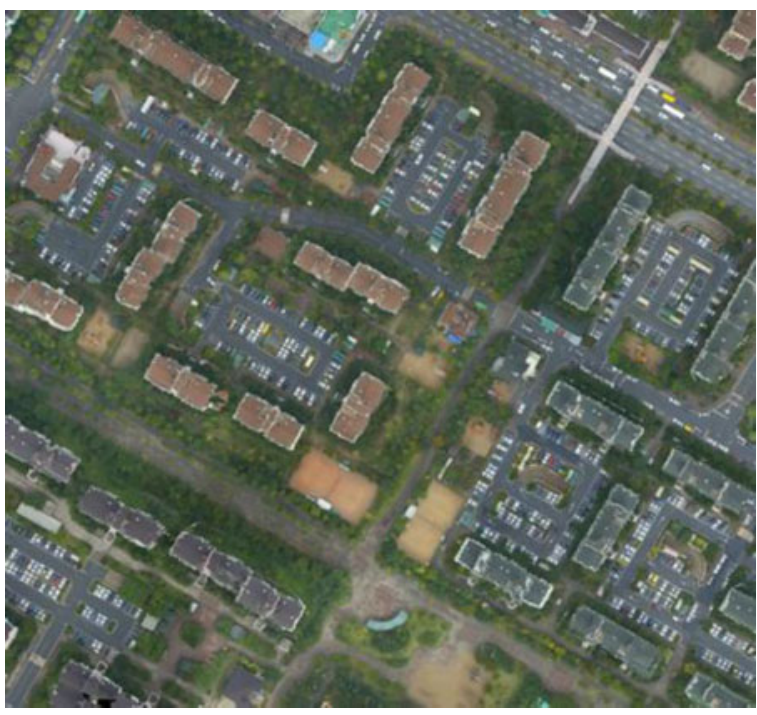

Fig. 23. True orthophoto mosaic (dataset 2).

and Th of $0.75 \mathrm{~m}$, and for dataset 2 the GSD is $9 \mathrm{~cm}$ and $\mathbf{T h}$ of $10 \mathrm{~m}$. The Th parameters were defined for this experiment (even though it was not necessary) to restrict the occlusion detection for only taller structures, which are just the main concern when dealing with occlusion.

The true orthophoto mosaics obtained in these experiments show a high completeness of occlusion detection and compensation, and regarding the seamlines, it is possible to note its mitigation (see Figs. 23 and 24). These results (true orthophoto mosaics) were achieved by the following processes: the occlusion detection by SGBM, the occlusion compensation by nearest ground nadir method, and the seamline mitigation by the feathering approach, thus obtaining a high-quality mosaic (mismatch assessment).

The results obtained by the SGBM show that the occlusion detection has high percentages of completeness. The occlusion compensation using the adjacent images fills the occlusion regions properly. It is worth noting that the feathering approach, applied after the Voronoi diagram generation, minimizes the effect of the seamlines to a sufficient degree. The time of processing also showed promising results when compared to the other methods.

The remaining radiometric variation in Fig. 24 is due to the high attitude variation of the UAV platform during the data acquisition, which caused a larger interference of the sunlight when compared to a more stable platform, such as the one used for acquiring dataset 2. An alternative to reduce this effect is to apply an image block radiometric adjustment. Another effect caused, when a DBM is not used, is the nonrefined borders of the buildings - which can be mitigated by using a high-density point cloud obtained either by LiDAR or dense image matching.

\section{Advantages and Disadvantages Compared to the State of the Art}

After explanation of the original proposed method and the assessment of results obtained in the experiments, it is important 


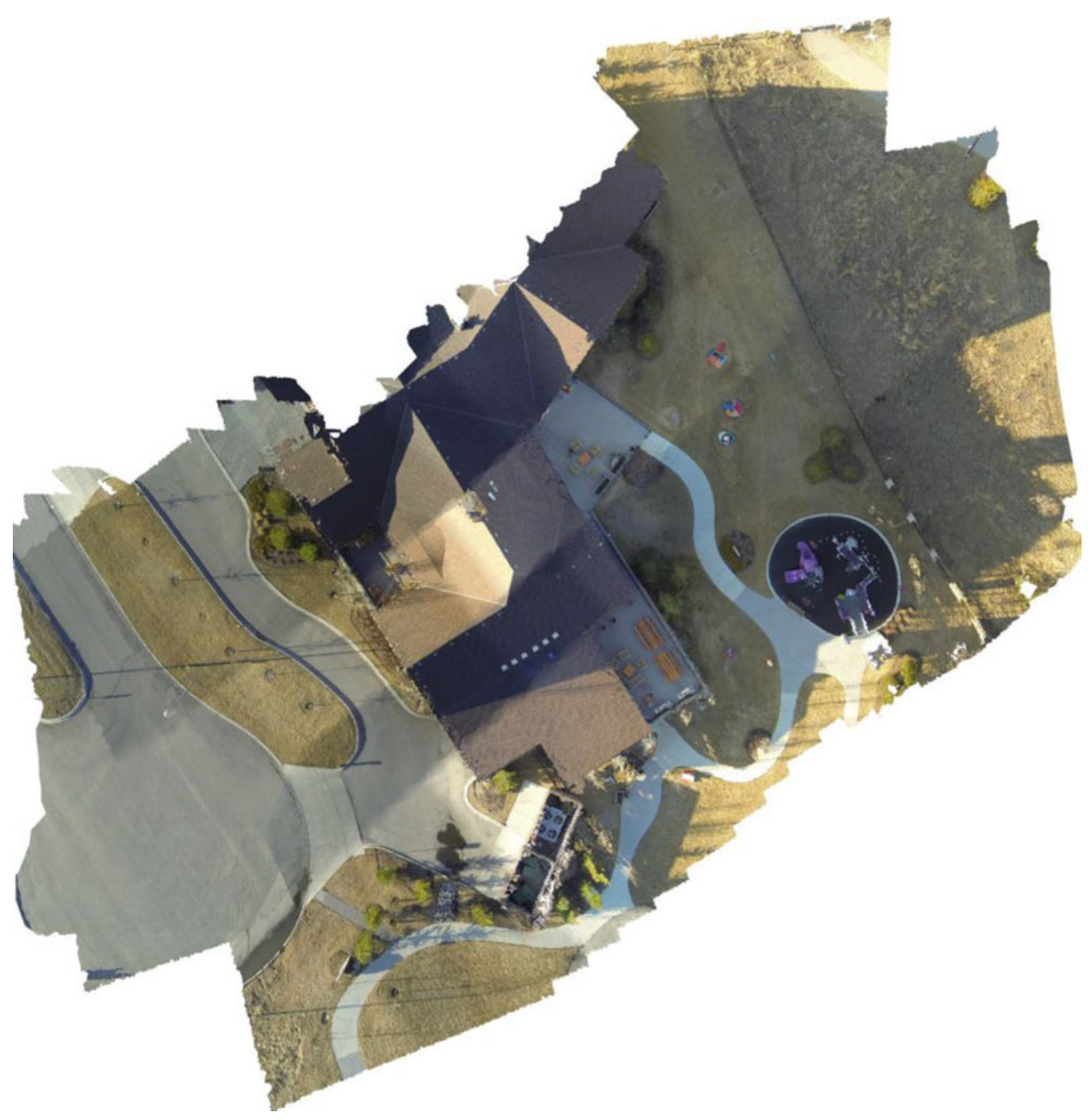

Fig. 24. True orthophoto mosaic (dataset 1).

to summarize its contribution compared to other state-of-the-art methods. The following topics detail the advantages of using the SGBM.

1) The main contribution of the SGBM is to enable the use of an irregularly spaced point cloud as input information - such as the ones used in the experiments. An initial interpolation step to obtain a gridded-DSM is not necessary, which speeds up the time of processing and avoids undesired artifacts in the surface representation. This characteristic of using a TIN over an original point cloud is not observed in any occlusion detection method presented in Section II (only also using a DBM).

2) As in the SGBM, a pixel-by-pixel analysis is not considered, the density of the input point cloud has no correlation with the desired spatial resolution for the output image (datasets 1 and 2 have nonuniform densities along the surface). The methods based on gridded-DSM require an interpolation of the DSM with the same spatial resolution as the output image. It generates a huge input dataset in case of high-resolution images.
3) The possibility of using a height threshold (Th) enables the user to define the structures that are intended to be considered (only tall buildings, for example), avoiding unnecessary computation processes, which are not seen in the other methods.

The negative aspect of the proposed method is the high frequency of revisit around the ground nadir point, due to the strategy for defining the radial directions. However, this can be seen in the majority of the methods in the state of the art and, indeed, does not affect the quality of the generated true orthophoto, just the processing time.

Although default values $(\Delta \boldsymbol{r}=\mathrm{GSD}$ and $\boldsymbol{T} \boldsymbol{h}=0 \mathrm{~m})$ is defined in our computational code, especially the $\boldsymbol{T} \boldsymbol{h}=0 \mathrm{~m}$ can produce a lot of occlusion segments, which can be undesired. The parameters $\Delta \mathbf{r}$ and $\mathbf{T h}$ also can be defined by the user, however, it is not considered a disadvantage because it is not a required action. Therefore, if it is intended to restrict the occlusion detection for specific heights, the user can set it, otherwise any negative height variation will be understood as a beginning of an occlusion. 


\section{CONCLUSION AND RECOMMENDATIONS FOR FUTURE WORK}

True orthophoto mosaics are important products to be used as base information for projects performed over urban areas. Although the principles can be considered as a simple approach, the generation is not a trivial procedure, as can be seen in the literature and this paper. Several steps must be accomplished to consider all elements above the ground during the true orthorectification process.

In this paper, a new approach for occlusion detection for true orthophoto mosaic generation is presented. The proposed procedure uses an irregularly spaced point cloud to identify occlusion areas, different from any other occlusion detection methods applied for aerial true orthophoto generation.

This original approach avoids the interpolation process as an initial step for occlusion detection, and consequently, the insertion of additional errors into the surface representation. Therefore, the use of a TIN as data structure for surface representation is the main contribution in this paper. Other contributions are: a better completeness and processing time when compared with state-of-the-art methods; the possibility of using different point cloud densities along the surface; and the option of defining the minimum height of objects to have their occlusions detected.

The results obtained by applying the SGBM, presented in Section IV, showed a high completeness percentage of occlusion detection (more than 90\%). The completeness values obtained by the proposed method have been better than other methods, particularly the Z-buffer. The geometric quality of the generated true orthophoto mosaics was also evaluated and good alignments can be observed (RMSE at the subpixel level). The processing time indicates the computational adequacy of the proposed method.

For future work, the implementation of different sweep methods is suggested for a more efficient radial exploitation, aiming the revisit reduction near the ground nadir point and the reduction of computation effort. By using a different sweep approach, it will be possible to apply the SGBM for datasets from pushbroom cameras, which only have occlusions along the across-track direction. Moreover, the application of other color correction procedures, such as an image block radiometric adjustment is recommended to refine the color variations on the true orthophoto mosaic. Another recommendation is the evaluation of the influence of using different point cloud density, over the DSM, during the occlusion detection for true orthophoto generation, and the assessment of buildings' border quality compared to a photogrammetric restitution.

\section{ACKNOWLEDGMENT}

The authors would like to thank the Department of Cartography (São Paulo State University, Brazil) and the DPRG (Digital Photogrammetry Research Group) from Purdue University, West Lafayette, IN, USA, for providing valuable facilities/resources, as well as Dr. C. Kim (Myongi University) for the supply of Z-buffer and Angle-based methods implementation to generate results for comparison.

\section{REFERENCES}

[1] P. R. Wolf and B. A. Dewitt, Elements of Photogrammetry - With Applications in GIS, 3rd ed. New York, NY, USA: McGraw-Hill, 2000, p. 608 .

[2] E. M. Mikhail, J. S. Bethel, and J. C. McGlone, Introduction to Modern Photogrammetry. New York, NY, USA: Wiley, 2001, p. 479.

[3] M. O. Nielsen, "True orthophoto generation," M.S. thesis, Informat. Mathe. Modelling, Tech. Univ. of Denmark, Lyngby, Denmark, 2004.

[4] A. F. Habib, E. Kim, and C. Kim, "New methodologies for true orthophoto generation," Photogrammetric Eng. Remote Sens., vol. 73, no. 1, pp. 25-36, 2007.

[5] F. Amhar, J. Jansa, and C. Ries, "The generation of true orthophotos using a 3D building model in conjunction with a conventional DTM," Int. Archives Photogrammetry Remote Sens., vol. 32, pp. 16-22, 1998.

[6] J. Y. Rau, N. Y. Chen, and L. C. Chen, "True orthophoto generation of built-up areas using multi-view images," Photogrammetric Eng. Remote Sens., vol. 68, pp. 581-588, 2002.

[7] Z. Qin, W. Li, M. Li, Z. Chen, and G. Zhou, "A methodology for true orthorectification of large-scale urban aerial images and automatic detection of building occlusions using digital surface model," in Proc. IEEE Int. Geosci. Remote Sens. Symp., 2003, pp. 729-731.

[8] G. Zhou, W. Chen, J. A. Kelmelis, and D. Zhang, "A comprehensive study on urban true orthorectification," IEEE Trans. Geosci. Remote Sens., vol. 43, no. 99, pp. 2138-2147, Sep. 2005.

[9] L. Chen, T. Teo, J. Wen, and J. Rau, "Occlusion-compensated true orthorectification for high-resolution satellite images," in Photogrammetric Rec., 2007, vol. 22, no. 117, pp. 39-52.

[10] E. Kwak and A. Habib, "Automatic representation and reconstruction of DBM from LiDAR data using recursive minimum bounding rectangle," ISPRS J. Photogrammetry Remote Sens., vol. 93, pp. 171-191, 2014.

[11] W. Schickler and A. Thorpe, "Operational procedure for automatic true orthophoto generation," Int. Achieves Photogrammetry Remote Sens., vol. 32, Part 4, pp. 527-532, 1998.

[12] L. Barazzetti, R. Brumana, D. Oreni, M. Previtali, and F. Roncoroni, “Trueorthophoto generation from UAV images: Implementation of a combined photogrammetric and computer vision approach," ISPRS Ann. Photogrammetry, Remote Sens. Spatial Inf. Sci., vol. II-5, pp. 57-63, 2014.

[13] J. Wu, S. Jie, W. Yao, and U. Stilla, "Building boundary improvement for true orthophoto generation by fusing airborne LiDAR data," in Proc. Ann. de Joint Urban Remote Sens. Event, 2011, pp. 125-128.

[14] X. Wang and J. Xie, "A method for true orthophoto generation based on projection and iteration strategy," ISPRS Ann. Photogrammetry, Remote Sens. Spatial Inf. Sci., vol. I-4, pp. 311-314, 2012.

[15] G. Zhou, Y. Wang, T. Yue, S. Ye, and W. Wang, "Building occlusion detection from ghost images," IEEE Trans. Geosci. Remote Sens., vol. 55, no. 2, pp. 1074-1084, Feb. 2017.

[16] K. I. Bang, A. F. Habib, S. W. Shin, and K. O Kim, "Comparative analysis of alternative methodologies for true orthophoto generation from high resolution satellite imagery," in Proc. ASPRS 2007 Annual Conf., 2007, pp. 34-45.

[17] R. Wimmer, "True orthophoto generation," Bachelor thesis, Landshut, Univ. of Applied Science, Berlin, Germany, 2010.

[18] H. C. Oliveira, M. Galo, and A. P. Dal Poz, "Height-gradient-based method for occlusion detection in true orthophoto generation," IEEE Geosci. Remote Sens. Lett., vol. 12, no. 11, pp. 2222-2226, Nov. 2015.

[19] D. F. Rogers, Procedural Elements for Computer Graphics, New York, NY, USA: McGraw-Hill, 1985, p. 422.

[20] H. C. Oliveira and M. Galo, "Occlusion detection by height gradient for true orthophoto generation, using LiDAR data," Int. Archives Photogrammetry, Remote Sens. Spatial Inf. Sci., vol. XL-1/W1, pp. 275-280, 2013.

[21] T. Peucker, R. Flower, J. Little, and D. Mark, "The triangulated irregular network," in Proc. Digit. Terrain Models Symp., 1978, pp. 96-103.

[22] B. Delaunay, "Sur la sphère vide," in Bull. de l'académiedessciences de l'urss, 1934, pp. 793-800.

[23] A. Wehr and U. Lohr, "Airborne laser scanning - An introduction and overview," ISPRS J. Photogrammetry Remote Sens., vol. 54, pp. 68-82, 1999.

[24] N. Paparoditis, C. Thom, and H. Jibrini, "Surface reconstruction in urban areas from multiple views of aerial digital frame cameras," Int. Archives Photogrammetry Remote Sens., vol. XXXIII, Suppl B3, pp. 43-50, 2000.

[25] H. Hirschmüller, "Accurate and efficient stereo processing by semi-global matching and mutual information," in Proc. IEEE Conf. Comput. Vision Pattern, 2005, vol. 2, pp. 807-814.

[26] M. Radwan and B. Makarovic, "Digital mono plotting systemImprovements and tests," in Proc. XIV Congr. Int. Soc. Photogrammetry, 1980, pp. 308-327. 
[27] H. C. Oliveira, A. F. Habib, A. P. Dal Poz, and M. Galo, "Height gradient approach for occlusion detection in UAV imagery," Int. Archives Photogrammetry, Remote Sens. Spatial Inf. Sci., 2015b, vol. XL-1/W4, pp. 263-268.

[28] Z. Lari and A. F. Habib, "A new approach for segmentation-based texturing of laser scanning data," Int. Archives Photogrammetry, Remote Sens. Spatial Inf. Sci., vol. XL-5/W4, pp. 115-121, 2015.

[29] P. J. Burt and E. H. Adelson, "A multiresolution spline with application to image mosaics," ACM Trans. Graph., vol. 2, no. 4, pp. 217-236, 1983.

[30] F. He, A. F. Habib, and A. Al-Rawabdeh, "Planar constraint for an improved UAV-image-based point cloud generation," Int. Archives Photogrammetry, Remote Sens. Spatial Inf. Sci., vol. XL-1/W4, pp. 269-274, 2015.

[31] C. Wiedemann, C. Heipke, H. Mayer, and O. Jamet, "Empirical evaluation of automatically extracted road axes," Empirical Evaluation Methods in Computer Vision. Washington, DC, USA: IEEE Computer Society Press, 1998, pp. 172-187.

[32] A. J. Fazan and A. P. Dal Poz, "Rectilinear building roof contour extraction based on snakes and dynamic programming," Int. J. Appl. Earth Observ. Geoinf., vol. 25, pp. 1-10, 2013.

[33] K. Oda, W. Lu, O. Ushida, and T. Doihara, "Triangle-based visibility analysis and true orthoimage generation," Int. Archives Photogrammetry, Remote Sens. Spatial Inf. Sci., vol. 35-Part3, pp. 623-628, 2004.

[34] W. Xiao, J. Wanshou, and B. Fuling, "Occlusion detection analysis based on two different DSM models in true orthophoto generation," in Proc. SPIE, vol. 7146, 2008, doi: 10.1117/12.813164.

[35] O. Faugeras, Three-Dimensional Computer Vision-A Geometric Viewpoint, Cambridge, MA, USA: MIT Press, 1999, p. 663.

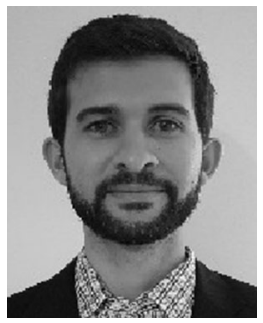

Henrique Cândido de Oliveira received the B. Sc. degree in cartographic engineering, the M. Sc. and Ph.D. degrees in digital photogrammetry from São Paulo State University, Presidente Prudente, Brazil, in 2010, 2013, and 2016, respectively.

He is currently a Professor of geomatics at the Faculty of Civil Engineering, Architecture, and Urban Planning, University of Campinas, Campinas, Brazil. His research interests are related to the field of digital photogrammetry, laser scanning, integration of lowcost sensors, image analysis, IoT for smart cities, and transportation.

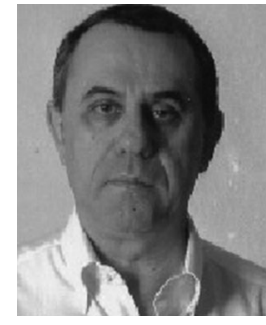

Aluir Porfírio Dal Poz received the B.Sc. degree in cartographic engineering from São Paulo State University, Presidente Prudente, Brazil, in 1987, the M.Sc. degree in geodetic science from Paraná Federal University, Curitiba, Brazil, in 1991, and the Ph.D. degree in transportation engineering from São Paulo University in 1996.

He is currently a Full Professor in the Department of Cartography, São Paulo State University. His expertise and current research interests include the areas of image analysis and digital photogrammetry.

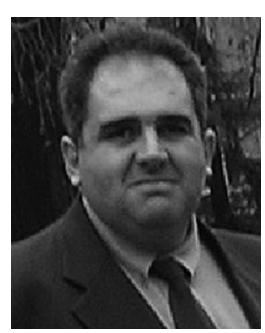

Mauricio Galo received the B.Sc. degree in cartographic engineering from UNESP - Universidade Estadual Paulista Júlio de Mesquita Filho, Presidente Prudente - SP, Brazil, in 1986; the M.Sc. degree in geodetic sciences from the UFPR - Federal University of Paraná, Curitiba, Brazil - PR, in 1993; and the Ph.D. degree in electric engineering from UNICAMP - State University of Campinas, Campinas, Brazil - SP, in 2003.

Currently, he is with the Department of Cartography, Universidade Estadual Paulista Júlio de Mesquita Filho, Presidente Prudente - SP as an Assistant Professor. His research interests include digital photogrammetry, digital camera calibration, airborne LASER scanning and feature extraction.

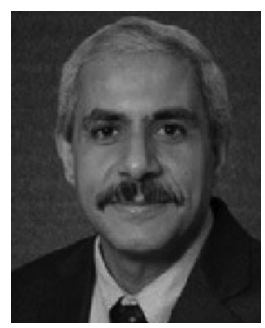

Ayman Fawzy Habib received the M.Sc. and Ph.D. degrees in photogrammetry from The Ohio State University, Columbus, OH, USA, in 1993 and 1994, respectively.

$\mathrm{He}$ is currently a Professor of geomatics at the Lyles School of Civil Engineering, Purdue University, West Lafayette, IN, USA. His research interests include the fields of terrestrial and aerial mobile mapping systems, modeling the perspective geometry of nontraditional imaging scanners, automatic matching and change detection, automatic calibration and stability analysis of low-cost digital cameras, utilizing low-cost imaging systems for infrastructure monitoring and biomedical applications, incorporating analytical and free-form linear features in various photogrammetric orientation procedures, object recognition in imagery, UAV-based 3-D mapping, as well as integrating photogrammetric data with other sensors/datasets (e.g., GPS/INS, GIS databases, multispectral sensors, and LiDAR). 